\section{AB1311-HPR RHEUMATIC AND MUSCULOSKELETAL DISEASES MANAGEMENT TOOL HELPS TO IMPROVE TREAT-TO- TARGET THERAPY AND PATIENTS' ADHERENCE TO TREATMENT}

P. Wu ${ }^{1}$, W. Li ${ }^{1}$, H. Zeng ${ }^{2}$, F. Lian ${ }^{2}{ }^{1}$ The First Affiliated Hospital of Sun Yat-Sen University, Dept. of Orthopedics, Guangzhou, China; ${ }^{2} T$ The First Affiliated Hospital of Sun Yat-Sen University, Rheumatology, Guangzhou, China

Background: Many of the rheumatic and musculoskeletal diseases (RMD) are long term, painful, and function affecting, which takes both the doctors and patients a lot of time and effort. The number of Rheumatologists are not sufficient for the huge population of RMD patients in China. The doctor patient ratio is as low as 1:1000. Relatively inadequate medical resources, traffic inconvenience in rural area, and patients' insufficient understandings of the RMD may cause delayed medical intervention and poor prognosis. Effective RMD patient management tools which provide disease monitoring and enough doctor-patient communication is essential to improve the patients' adherence to treatment. We designed an RMD management app according to the social, cultural and economic situation of Chinese patients, which helps to facilitate shared decision making and relieve the pressure of insufficient medical resources.

Objectives: We aim to investigate the effect of RMD patient management app on treat-to-target therapy and patients' adherence and satisfaction to treatment.

Methods: An observational survey was administrated using a RMD patient management app. The app was designed and improved by Rheumatologist, orthopedics, nurses, patients, and app technical experts. Patients were offered with a questionnaire in regard to satisfaction with the app and their attitudes about the disease. General therapeutic principles, rehabilitation exercise videos and follow-up information were distributed through the app. Warning signals were sent whenever there was a flag sign of exacerbation. The demographic and clinical data, social and economic status, and drug retention rates of the patients were documented. The survey was designed by clinical experts from relevant departments and developed by both doctors and patients.

Results: All patients were supervised by the rheumatologist and orthopedist when using the app. In all the patients included, there were cases of rheumatoid arthritis (35.3\%), osteoarthritis (32.4\%), ankylosing spondylitis $(26.5 \%)$, and other chronic arthritis (5.8\%). The mean age $38.5 \pm 15.8$ years old, with $52.9 \%$ male and $47.1 \%$ female. Most of the patients ( $85.3 \%$ ) believed that the app was helpful. Young patients were more likely to respond to the survey than older patients. Some patients $(79.4 \%)$ had increased compliance because the app offered more chances to communicate with the doctors, which increase their understanding and confidence about the disease. Three patients received flag signs of exacerbation much earlier than they could get to the hospital. From the feedback of the patients, we realized that the patient would like to have more information to keep them from stepping on the trap of false advertisement for therapy (which is very commonly seen in China).

Conclusion: RMD patient need to manage disease activity, daily function and mental state. Insufficient medical resources and patients' knowledge about the disease may lead to poor adherence and prognosis. RMD patient management tool on app was a feasible and cost-effective approach for data collection and patient education. The app increased treat-to-target therapy and patients' adherence to treatment.

References: None.

Disclosure of Interests: None declared

DOI: 10.1136/annrheumdis-2020-eular.2245

\section{AB1312-HPR A COMPARISON OF THE EFFECTIVENESS OF TWO DIFFERENT KINESIO TAPE APPLICATIONS ON PAIN, FATIGUE AND HEALTH STATUS IN WOMEN WITH FIBROMYALGIA}

S. Toprak Celenay ${ }^{1}$, B. Anaforoglu Kulunkoglu' ${ }^{1}$, O. Mete ${ }^{1} .{ }^{1}$ Department of Physiotherapy and Rehabilitation, Health Sciences Faculty, Ankara Yildirim Beyazit University, Ankara, Turkey

Background: Fibromyalgia (FM) is a syndrome characterized mainly by chronic widespread pain, fatigue and a decrease in health status. Kinesio tape (KT), one of complementary approaches, has favorable effects on clinic findings in FM, but studies investigating which types of the KT applications are more effective in FM are scarce.

Objectives: This study aimed to compare the effectiveness of fascial correction and FanCut KT applications on pain, fatigue and health status in women with FM. Methods: A total of 27 women with FM were included, allocated into fascial correction (Group 1) (n:14, age: 41.50 (30.25) years, body mass index (BMI): 24.85 $(5.28) \mathrm{kg} / \mathrm{m}^{2}$ ) and FanCut techniques (Group 2) (n:13, age: 45.00 (21.00) years,
BMl: $25.60(4.55) \mathrm{kg} / \mathrm{m}^{2}$ ) groups. The fascial correction technique performed with approximately $25 \%$ to $50 \%$ of tension and oscillated in longitudinal direction on overall back was used in the Group 1; while, the FanCut technique performed with $0 \%$ tension on the overall back was used in the Group 2. Exercise program was carried out 2 days a week for 6 weeks under the supervision of a physiotherapist in both groups. Pain intensity and severity of fatigue with the Visual Analog Scale and health status with the Fibromyalgia Impact Questionnaire were evaluated at the baseline and after the 6-weeks treatment.

Results: Physical characteristics of the groups were similar $\left(\mathrm{p}_{\mathrm{age}}=0.190, \mathrm{p}_{\mathrm{bmi}}=0.808\right)$. After the treatment, it was found that pain intensity $(p=0.001 ; p=0.001)$ and the severity of fatigue $(p=0,001 ; p=0.003)$ decreased and health status $(p=0.001 ; p=0.002)$ improved in both the group 1 and 2, respectively. Moreover, fatigue decreased $(p=0.008)$ and health status improved $(p=0.021)$ in the group 1 in comparison to the group 2; but pain intensity $(\mathrm{p}=0.085)$ did not differ between the groups.

Conclusion: In this study, it was observed that both the fascial correction and the FanCut KT applications were effective in decreasing pain and fatigue, and improving the health status of women with FM. In addition, the study suggested that the fascial correction KT application was superior in decreasing fatigue and improving health status in comparison to the FanCut $\mathrm{KT}$ application in women with FM. In clinics, the KT application may be considered as a non-pharmacologic and complementary therapy to improve the symptoms of FM. Moreover the fascial correction KT application may be more effective in improving some parameters due to fascia dysfunction in FM.

\section{References:}

[1] G.V. Espí-López, M. Inglés, A.C. Ferrando, P. Serra-Añó, Effect of Kinesio taping on clinical symptoms in people with fibromyalgia: A randomized clinical trial, J. Back Musculoskelet. 32 (4) (2019) 561-567

[2] K. Kase, J. Wallis, T. Kase, Clinical therapeutic applications of the Kinesio taping method, Kení-kai information, Tokyo, Japan, 2003.

Disclosure of Interests: None declared

DOI: 10.1136/annrheumdis-2020-eular.790

\section{AB1313-HPR EFFECTS OF N-ACETYLCYSTEINE ON PULMONARY FUNCTIONS IN PATIENTS WITH SYSTEMIC SCLEROSIS: A DOUBLE BLIND, PLACEBO CONTROLLED STUDY}

M. A. Nazarinia ${ }^{1,2}$, S. Mehrabi ${ }^{3}$, Z. Khodamoradi ${ }^{4}$, M. Mazidi Moradi ${ }^{3}{ }^{1}$ Shiraz University of Medical Sciences, Shiraz, Iran, Division of Rheumatology, Department of Internal Medicine, Namazee Hospital, Shiraz, Iran (Islamic Republic of); ${ }^{2}$ Shiraz University of Medical Sciences, Shiraz, Iran, Shiraz Geriatric Research Center, Shiraz, Iran (Islamic Republic of); ${ }^{3}$ Shiraz University of Medical Sciences, Shiraz, Iran, Department of Internal Medicine, Namazee Hospital, Shiraz, Iran (Islamic Republic of); ${ }^{4}$ Shiraz University of Medical Sciences, Shiraz, Iran, Student Research Committee, Shiraz, Iran (Islamic Republic of)

Background: Systemic sclerosis (SSc) is a systematic and rare autoimmune disease that affects many organs. $\mathrm{N}$-acetylcysteine (NAC), thiol containing compound, can act both as the precursor of reduced glutathione and direct scavenger of reactive oxygen species.

Objectives: We assessed the clinical effect of NAC on pulmonary function test of patients with diffuse scleroderma.

Methods: This study is a randomized double blind clinical trial that was done on 25 patients with diffuse SSc without lung involvement on primary chest high-resolution computed tomography. Placebo was administered for 13 patients and 1200 milligram NAC for 12 patients. Body plethysmography parameters were assessed at the beginning of the study and after 24 weeks.

Results: Patients in the two groups were matched in the basic demographic data like age, duration of disease, and modified Rodnan skin score. The analysis showed no significant differences in parameters of plethysmography between the two groups. After importing the data of 2 patients in the placebo-treated group, who developed interstitial lung disease, DLCO in the placebo-treated group was $90.69 \pm 21.29$ milliliter at the end of the study, which significantly decreased compared with the beginning of the study $(102.30 \pm 13.83 \mathrm{ml})$. Also, changes of DLCO between the two groups were significantly different.

Conclusion: In this trial, sensitivity of DLCO as the first marker in evaluation of pulmonary function in patients with SSc was confirmed. On the other hand, NAC had no effect versus placebo in a period of 24 weeks. We recommend that more studies with larger sample size and longer duration should conduct for further evaluation. References: No reference.

Acknowledgments: The present article was extracted from the thesis written by Maryam Mazidi Moradi. The authors would like to thank Shiraz University of Medical Sciences, Shiraz, Iran and also Center for Development of Clinical Research of Nemazee Hospital and Dr. Nasrin Shokrpour for editorial assistance.

Disclosure of Interests: None declared

DOI: 10.1136/annrheumdis-2020-eular.3032 